\title{
Detection and characterization of epistasis between QTLs on plant height in rice using single segment substitution lines
}

\author{
Haitao Zhu, Ziqiang Liu, Xuelin Fu, Ziju Dai, Shaokui Wang, Guiquan Zhang, Ruizhen Zeng and Guifu Liu* \\ State Key Laboratory for Conservation and Utilization of Subtropical Agro-Bioresources, Guangdong Key Laboratory of Plant Molecu- \\ lar Breeding, College of Agriculture, South China Agricultural University, Guangzhou 510642, P. R. China
}

\begin{abstract}
Hua-jing-xian 74 and its 12 single segment substitution lines (SSSLs) in rice were used as crossing parents to construct a half diallel crossing population. A total number of 91 materials were grown under three planting densities. By analysis of average plant height $(\mathrm{PH})$ over all environments 10 SSSLs were detected with significant additives and 6 SSSLs with significant dominances. These SSSLs were further tested under different densities respectively, indicating that some of single locus effects were sensitive to densities and the conditions under the density of $16.7 \mathrm{~cm} \times 16.7 \mathrm{~cm}$ maybe inhibited the expressing of these PH QTLs. Qualitative and quantitative analyses of each four participating genotypes indicated that digenic interactions among these QTLs were prevalent. Of 66 tested interactions, about 42.4\% were epistatic (P $<5 \%$ ). Although some QTLs hadn't single locus effects, they were possible to form digenic interactions. A significant finding was that the detected epistases were mostly negative. Additionally, these epistases were also found being sensitive to planting densities, the conditions under the density of $10 \mathrm{~cm} \times 16.7 \mathrm{~cm}$ perhaps promoted the expressing of epistatic interactions among PH QTLs.
\end{abstract}

Key Words: epistasis, QTL, single segment substitution line, plant height, rice.

\section{Introduction}

Plant height in rice is one of the most important agronomic traits. Most high-yielding varieties had the features to reduce plant height with high lodging resistance, favorable plant type, and high-harvest index in almost all rice-growing countries (Ming 1987). On the other hand, plant height is highly associated with biomass that is positively correlated with grain yield. In the past, great efforts have been made to dissect the genetic basis of plant height. Researches indicated that plant height in rice is generally controlled by both qualitative and quantitative genes. More than 1000 QTLs on plant height have been mapped in rice chromosomes (http:// www.gramene.org/qtl). While the action mechanism of multiple gene combination is mainly accumulation and interaction of single gene effects, that is so-called additive effect and dominant or epistatic effect. Apparently, epistatic interaction is one of important genetic factors on quantitative traits as $\mathrm{PH}$ etc.

For genes affecting quantitative traits, epistasis had been defined as the deviation from the sum of the independent effects of the individual genes (Falconer 1989). The univer-

Communicated by Q. Qian

Received July 25, 2014. Accepted February 8, 2015.

*Corresponding author (e-mail: guifuliu@scau.edu.cn) sality and the importance of epistasis existed in the genetic system on quantitative traits had been proved by numerous classical studies (Cockerham and Zeng 1996, Cooper and Hammer 1996, Paterson et al. 1991). Epistatic effects between QTLs for many quantitative traits had also been widely estimated by using conventional experimental materials (Cho et al. 2007, Gao et al. 2007, Li et al. 1997, 2001, 2003, Li et al. 2008, Semel et al. 2006, Wang et al. 1999, Xiao et al. 1995, Zhao et al. 2010, 2013, Zhu et al. 2011, Zhuang et al. 1997). However it should be noticed that such applied populations segregating for an entire genome have different genetic backgrounds among lines or individuals, resulting in that estimations of QTL effects were confound with other genetic factors (Eshed and Zamir 1995). Tanksley (1993) suggested to develop novel materials as introgression lines (ILs) or near-isogenic lines (NILs) to clarify the nature of QTLs, in which most genetic variation is associated with the studied QTLs and detected epistasis more frequently (Ashikari and Matsuoka 2006, Eshed and Zamir 1995, 1996, Keurentjes et al. 2007). Eshed and Zamir (1995) presented a novel population consisting of 50 ILs in tomato and revealed at least 23 QTLs for total soluble solids content and 18 for fruit mass. In 1996, they sequentially analyzed interactions among these QTLs via 10 single heterozygotes and 45 double heterozygotes, and detected that the frequency of dominance $\times$ dominance epistasis was high 
(Eshed and Zamir 1996). In rice, by using these materials a mass of QTLs and their epistases on traits as heading date, plant height, yield and yield components etc were well analyzed (Lin et al. 2000, Tian et al. 2006, Wan et al. 2003, Xi et al. 2006, Yamamoto et al. 2000, Yano et al. 1997, 2000, 2001). To evaluate digenic interaction between pairs of QTLs, Yano's research team revealed additive $\times$ additive epistatic interactions among 8 QTLs on heading date in rice via developing double QTL near-isogenic lines (Lin et al. 2000, Yamamoto et al. 2000, Yano et al. 2000).

In our lab a library of 1596 single segment substitution lines (SSSLs), each carrying a single chromosomal segment derived from different donors in an otherwise homogeneous background of HJX74, was constructed (Xi et al. 2006, Zhang et al. 2004) and widely applied to detect QTLs affecting important agronomic traits in rice (He et al. 2005a, 2005b, 2005c, Xi et al. 2006). Since 2008 we focused on rice tiller number to reveal the feature of spatio-temporal expression of QTLs (Liu et al. 2009, 2010, 2012, Zhao et al. 2008), and also paid attention on the genetic study of panicle number, the effective tiller number at final developmental stage in rice (Liu et al. 2008). In 2012, 12 SSSLs were applied to detect epistases between pairs of QTLs on tiller number in rice and the result indicated that their epistatic interactions occurred were often and dynamic (Liu et al. 2012). In this paper the 12 SSSLs were again applied to assay for the epistatic interactions between pairs of QTLs on $\mathrm{PH}$. The result would further confirm the universality and the importance of epistasis between QTLs, and provide useful information for improving PH via heterosis and QTL pyramiding in rice.

\section{Materials and Methods}

\section{Plant materials}

Hua-jing-xian 74 (HJX74) and its 12 SSSLs were selected as experimental materials in this study (Table 1). They were first crossed each other to have constructed a $13 \times 12$ half diallel crossing combinations in 2008 , and then a total of 91 materials including 13 parents (HJX74 and the 12 SSSLs), 12 combinations of HJX74 $\times$ SSSL (in the single heterozygous state), and $66(12 \times 11 / 2) \mathrm{SSSL}_{\mathrm{i}} \times \mathrm{SSSL}_{\mathrm{j}}$ (in the dual heterozygous state), were grown in the field in 2009. Where the subscripts $i$ or $j$ was the serial number of SSSL ( $i$ is smaller than $j, i$ is from 1 to 11 and $j$ from 2 to 12). The recipient HJX74 is an elite indica variety from South China, and various donor varieties for SSSLs were collected worldwide. Each of the 12 SSSLs had only one donor segment substituted into HJX74 genetic background (Xi et al. 2006, Zhang et al. 2004). It was confirmed by molecular markers that the homozygous SSSL was crossed to HJX74 and to each of the other lines to generate introgression line hybrids heterozygous for single introgressions and for pairs of introgressions, respectively. Some background information about these SSSLs is also shown in Table 1.

\section{Field trials and evaluation of $\mathrm{PH}$}

Phenotype experiments were similar to our previous studies (Liu et al. 2008, 2009, 2010, 2012). Trials were conducted at the experimental station in South China Agricultural University, Guangzhou, China (at $\sim 113^{\circ}$ east longitude and $\sim 23^{\circ}$ north latitude). All 91 materials mentioned above were grown in spring season (duration from March to July) in 2009. Germinated seeds were sowed in a seedling bed, and seedlings were transplanted to a paddy field 20 days later with one plant per hill spaced under three planting densities $10 \mathrm{~cm} \times 16.7 \mathrm{~cm}(\mathrm{~d} 1), \quad 16.7 \mathrm{~cm} \times 6.7 \mathrm{~cm} \mathrm{(d2)} \mathrm{and}$ $23.3 \mathrm{~cm} \times 6.7 \mathrm{~cm}(\mathrm{~d} 3)$, respectively. Since the actual planting density adopted usually in production practice of rice in South China was $16.7 \mathrm{~cm} \times 16.7 \mathrm{~cm}$, in this study these three different densities approaching the actual density were set as experimental levels of density factors that affects apparently PH in rice. In each trial a completely randomized design was adopted, in which each plot consisted of four rows with 10 plants each row. The trials performed accordance management of the field with local standard practices. $\mathrm{PH}$ per hill was measured in 12 central individuals of each

Table 1. Single segment substitution lines (SSSL) of HJX74 and their codes, chromosomes (Chr.), length (cM), marker intervals and donors

\begin{tabular}{|c|c|c|c|c|c|}
\hline SSSL & Chr. & Putative QTL ${ }^{a}$ & Length (cM) & Marker interval & Donor \\
\hline \multicolumn{6}{|l|}{ HJX74 } \\
\hline W11-15-09-03 & 2 & Ph2-1 & 12.5 & RM112--RM213 & Basmati 370 \\
\hline W27-14-01-09-18 & 2 & $P h 2-2$ & 23.1 & RM526--RM425 & IAPAR9 \\
\hline W20-20-05-19-07 & 3 & Ph3-1 & 20.4 & RM168--RM571 & Chenglongshuijingmi \\
\hline W20-20-05-05-11 & 3 & Ph3-2 & 6.0 & RM135--RM55 & Chenglongshuijingmi \\
\hline W08-16-03-59 & 3 & $P h 3-3$ & 17.2 & PSM304--RM545 & IR64 \\
\hline W15-05-07-15 & 6 & Ph6-1 & 8.8 & Rm508--Rm225 & American jasmine \\
\hline W17-10-07-5-12 & 6 & Ph6-2 & 8.5 & RM133--RM587 & Ganxiangnuo \\
\hline W08-09-05-03 & 6 & Ph6-3 & 28 & RM508--RM549 & IR64 \\
\hline W19-18-9-6 & 7 & $P h 7$ & 24.5 & RM51--RM214 & Kyeema \\
\hline W17-46-40-10-07-04 & 8 & Ph8 & 8.3 & RM515--RM210 & Ganxiangnuo \\
\hline W02-17-06-15 & 9 & $\operatorname{Ph} 9$ & 17.0 & RM105--RM278 & Amol 3 \\
\hline W08-15-06-04-04 & 12 & Ph12 & 2.6 & RM235--RM17 & IR64 \\
\hline
\end{tabular}

${ }^{a}$ QTL was nominated by $P h$ followed by the chromosomal number. Additional number was given as more than one QTL was located in the same chromosome. QTL Ph2-1, for example, indicated the first QTL on plant height detected on chromosome 2. 
plot at maturity in rice. Plot averages were as basic units in statistical analysis in this paper.

\section{Statistical analysis}

Statistical model $y_{h k}=\mu+G_{h}+D_{k}+\varepsilon_{h k}$ was used for analysis of variance (ANOVA) to all data of 91 materials. Where $\mu$ was population mean value, $G$ and $D$ were genotypic effect and density effect, respectively, and $\varepsilon$ was the residual error. All components were assumed as random effects except for $\mu$ and $G$ that were fixed. To confirm the existence of individual QTL, we estimated the additive effect $(a)$ of QTL by (SSSL - HJX74) and the dominant effect $(d)$ by $(S S S L \times H J X 74-H J X 74)$ on the mean value of PH. Their significances were tested by the least significant difference (LSD) method with the statistics $L S D_{\alpha}=t_{a} \sqrt{\frac{2 M S_{e}}{n}}$, where $M S_{e}$ was the experimental error with the degree of freedom $d f_{e}=(k-1)(n-1), k$ and $n$ were the numbers of all genotypes and densities, respectively, and $t_{a}$ was the critical $t$-value under $d f_{e}$ degree of freedom and $\alpha$ probability level. To evaluate epistatic interaction between pair of QTL, a two-way ANOVA was independently performed on the four participating genotypes including, $H J X 74, S S S L_{i} \times H J X 74$, $S S S L_{j} \times H J X 74$ and $S S S L_{i} \times S S S L_{j}$. The genotypic value $G_{i j}$ could be divided into its components as follows, $G_{i j}=$ $\mu+d_{i}+d_{j}+e_{i \times j}$, where $\mu$ was equal to the PH mean value of $H J X 74, d_{i}$ or $d_{j}$ was the dominant effect of heterozygous $S S S L_{i} \times H J X 74$ or $S S S L_{j} \times H J X 74$. Then the epistatic interaction between $Q T L_{i}$ and $Q T L_{j}$ was estimated by $e_{i \times j}=$ $G_{i j}-\mu-d_{i}-d_{j}$. Its significance was tested by LSD method with $L S D_{\alpha}=t_{a} \sqrt{\frac{4 M S_{e}}{n}}$, where all parameters were the same with those mentioned above. ANOVA was carried out with the PROC GLM module of SAS (SAS Institute 1988).

\section{Results and Analysis}

\section{Detection of SSSLs with PH QTLS}

In spring season in 2009, the control of HJX74, the 12 SSSLs and their crossing combinations were grown in the field with the three different planting densities (d1, d2 and d3) in order to detect whether these SSSLs were with PH QTLs. Based on the averages over all experimental environments, both additive effects and dominant effects of these SSSLs were estimated and their significances were tested (Table 2). Of 12 SSSLs, there were 10 and 6 SSSLs to be detected with significant additives and dominances, respectively. All QTLs except for Ph12 were with significant single locus effects (additive and/or dominance). Except for the additives of QTLs Ph3-2 and Ph6-1, all single locus effects estimated on these QTLs were positive, indicating that these donor segments on SSSLs carried with synergistic genes for target trait. On the contrary, allele genes on QTLs Ph3-2 and Ph6-1 derived from donors would decrease PH by $4.7 \mathrm{~cm}$ and $5.6 \mathrm{~cm}$ when they were in a homozygous state, respectively.

\section{Characterization of these QTLS}

To deepen understanding of the characterization of QTL expressing, we estimated single locus effects of these QTLs under the three planting densities, respectively (Table 2). Under all experimental environments of the three densities, 12 QTLs were detected all with significant additives and/or dominances, implying that QTL expressing differed across density environments. Although most QTLs were with consistent effect directions, their effect estimation values differed greatly across the densities. The ranges of QTL effects (the maximum minus the minimum) among the three densities can be used to illustrate the degree of environmental sensitivity (DES) (Lin et al. 2000). The estimations of these ranges for the 12 QTLs changed from $0.6 \mathrm{~cm}$ to $11.9 \mathrm{~cm}$, indicating their DES (Table 2). The ranges of 5 QTL additives (on $P h 3-2, P h 6-2, P h 6-3, P h 7$ and $P h 9$ ) and 4 QTL dominances (on $P h 3-2, P h 3-3, P h 6-2$ and Ph12) were less than 4.0, suggesting that they were environmental-stable. The others could be considered as environmental sensitive. Under the density of $\mathrm{d} 2$ the QTL number detected was the fewest among the three densities, indicating that $\mathrm{d} 2$ conditions maybe inhibited the expressing of these PH QTLs.

\section{Detection of epistatic interactions}

To clarify the epistatic interaction between pair of QTLs, we polymerized two substitution segments into a hybrid via construction of crossing combination between two SSSLs. A half of diallel crossing population was made with the 12 SSSLs as parents to form a total number of $12 \times 11 / 2=66$ combinations. Confirmed by molecular markers, each crossing combination was heterozygous only at regions of two substitution segments. Apparently, these hybrids amounted to NILs with two PH QTLs. We then compared the average $\mathrm{PH}$ of different genotypic values including $H J X 74$, $S S S L_{i} \times H J X 74(S i), S S S L_{j} \times H J X 74(S j), S S S L_{i} \times \operatorname{SSSL}_{j}\left(S_{i j}\right)$. Fig. 1 shows the average PH for each four genotypes with active points at each QTL pair and with lines to link two adjacent nodes. For example, four genotypic values were in turn $92.5 \mathrm{~cm}(H J X 74), 97.6 \mathrm{~cm}(\mathrm{Si}), 99.7 \mathrm{~cm}(\mathrm{Sj})$ and $98.1 \mathrm{~cm}(\mathrm{Sij})$ when $i$ equaled 1 and $j$ did 2 . Then epistatic interaction between two QTLs could be roughly judged by evaluating the differences among these four genotypic values. If the distance of two points represented respectively genotypic values between $S i j$ and $S j$ differed apparently from that between $S i$ and $H J X 74$, indicating that the expressing of QTL on $S i$ was regulated by the QTL on $S j$, the digenic interaction could exist between the two QTLs. Otherwise epistasis didn't exist when these distances were approximatively consistent. As shown in Fig. 1, QTL on $S 1$ interacted with QTLs on all other SSSLs except for $S 10$. Since the distance between $S 1 \cdot 10$ and $S 10$ kept nearly consistent with that between $S 1$ and $H J X 74$, it implied that QTL on $S 1$ was independent of QTL on $S 10$.

\section{Estimation of epistatic effects}

To statistically confirm the existence of digenic interactions 
Table 2. QTL effects estimated under different density conditions

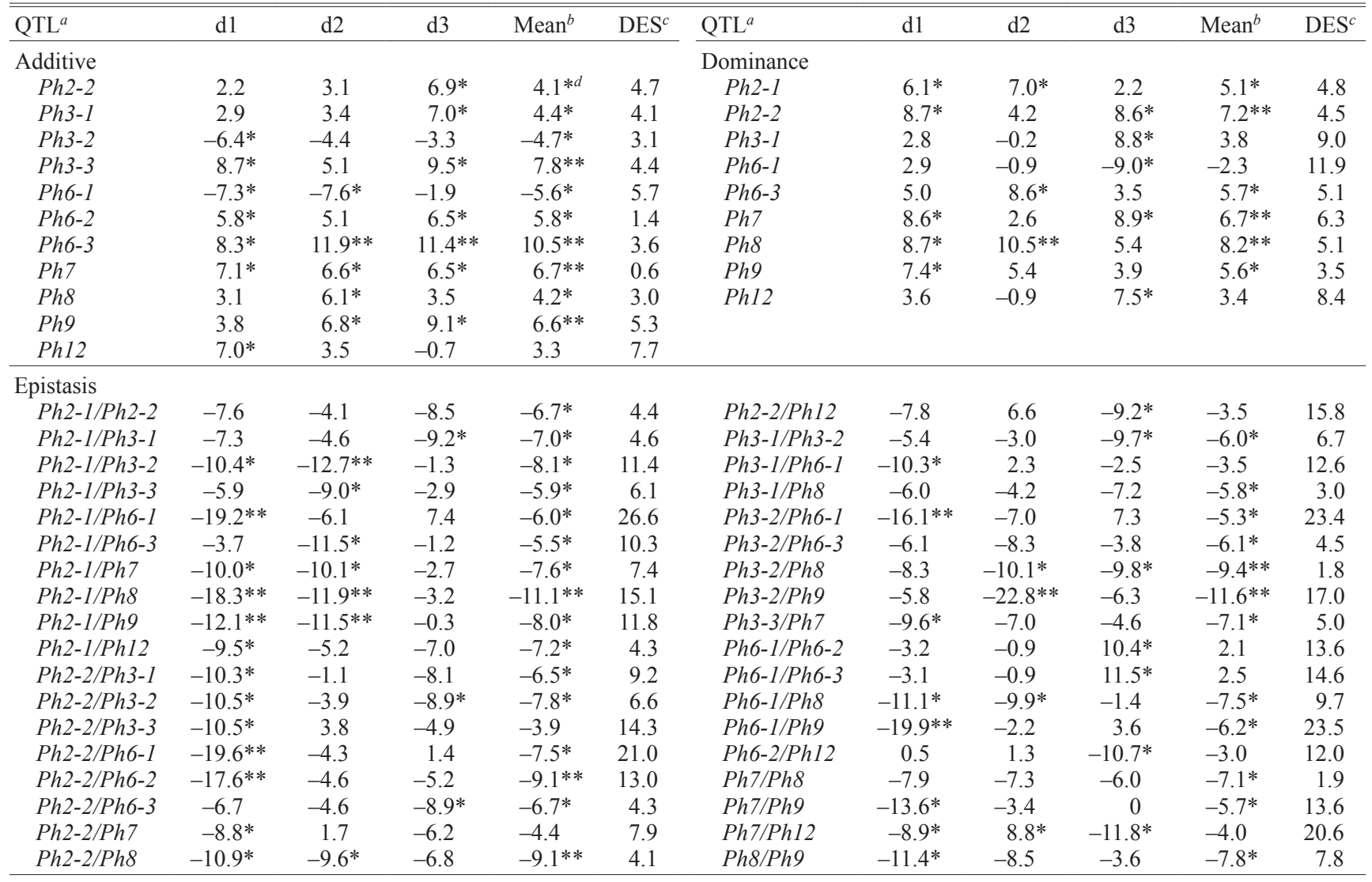

${ }^{a}$ QTL was nominated by $P h$ followed by the chromosomal number. Additional number was given as more than one QTL was located in the same chromosome. QTL Pn2-1, for example, indicated the first QTL on panicle number detected on chromosome 2.

${ }^{b}$ Mean represented a comprehensive condition averaged over all planting densities. $\mathrm{d} 1, \mathrm{~d} 2$ and $\mathrm{d} 3$ were the three planting densities, $10 \mathrm{~cm} \times 16.7 \mathrm{~cm}, 16.7 \mathrm{~cm} \times 16.7 \mathrm{~cm}$ and $23.3 \mathrm{~cm} \times 16.7 \mathrm{~cm}$, respectively.

${ }^{c}$ The degree of environmental sensitivity (DES) was calculated by the maximum minus the minimum among the estimations under the three densities.

${ }^{d}$ Numbers showed were QTL effects estimated, which positive or negative signs indicated that the alleles derived from donors increased or decreased plant height per plant compared with the control of HJX74, and which * or ** showed the significances at the probability of 0.05 or 0.01 .

among the 12 QTLs, we estimated epistatic effects between any two QTLs by the formula of $S S S L_{i} \times S S S L_{j}+H J X 74$ $-S S S L_{i} \times H J X 74-S S S L_{j} \times H J X 74$ on the averages of PH (Table 2). These estimations represented dominancedominance epistatic interactions between two QTLs. A total number of 28 pairs of QTLs was detected with significant epistatic effects at the level of $\mathrm{P}<0.05$, occupying $42.4 \%$ of 66 pairs of QTL combinations tested. This result was similar to the finding that there were $20-42 \%$ of the 45 dichromosome segment combinations to be epistatic in tomato and supported the suggestion that epistatic interactions between QTLs occurred at a high frequency (Eshed and Zamir 1996). Although the dominant effects of QTLs as Ph3-1, Ph3-2, Ph3-3, Ph6-1, Ph6-2 and Ph12 were insignificant, their interaction effects with other QTLs reached the significant level of $\mathrm{P}<0.05$. The result indicated that epistatic interaction between QTLs is also possible even though their independent effects are small or insignificant. Opposite with the additive effects and dominant effects detected, the epistatic effects were almost negative. The result implied that the epistatic interactions between QTLs tended to decrease $\mathrm{PH}$. The tendency for epistatic interactions to decrease target traits seemed a common phenomena (Eshed and Zamir 1996).

\section{Characterization of epistatic effects}

To character digenic interaction among these 12 QTLs, we also estimated their epistatic effects under the three planting densities, respectively (Table 2). A total of 32 pair QTLs were detected with significant epistatic effects at least under one of the three densities. This result further confirmed the universality of epistatic interactions between QTLs. However, only at certain conditions were these epistatic interactions detectable. No epistatic interactions could be detected simultaneously at the three densities. It seemed that epistasis was more easily detected at high density (like d1) than at low density (like $\mathrm{d} 2$ and $\mathrm{d} 3$ ). For instance, there were 21, 11 and 10 pairs of QTLs to be 

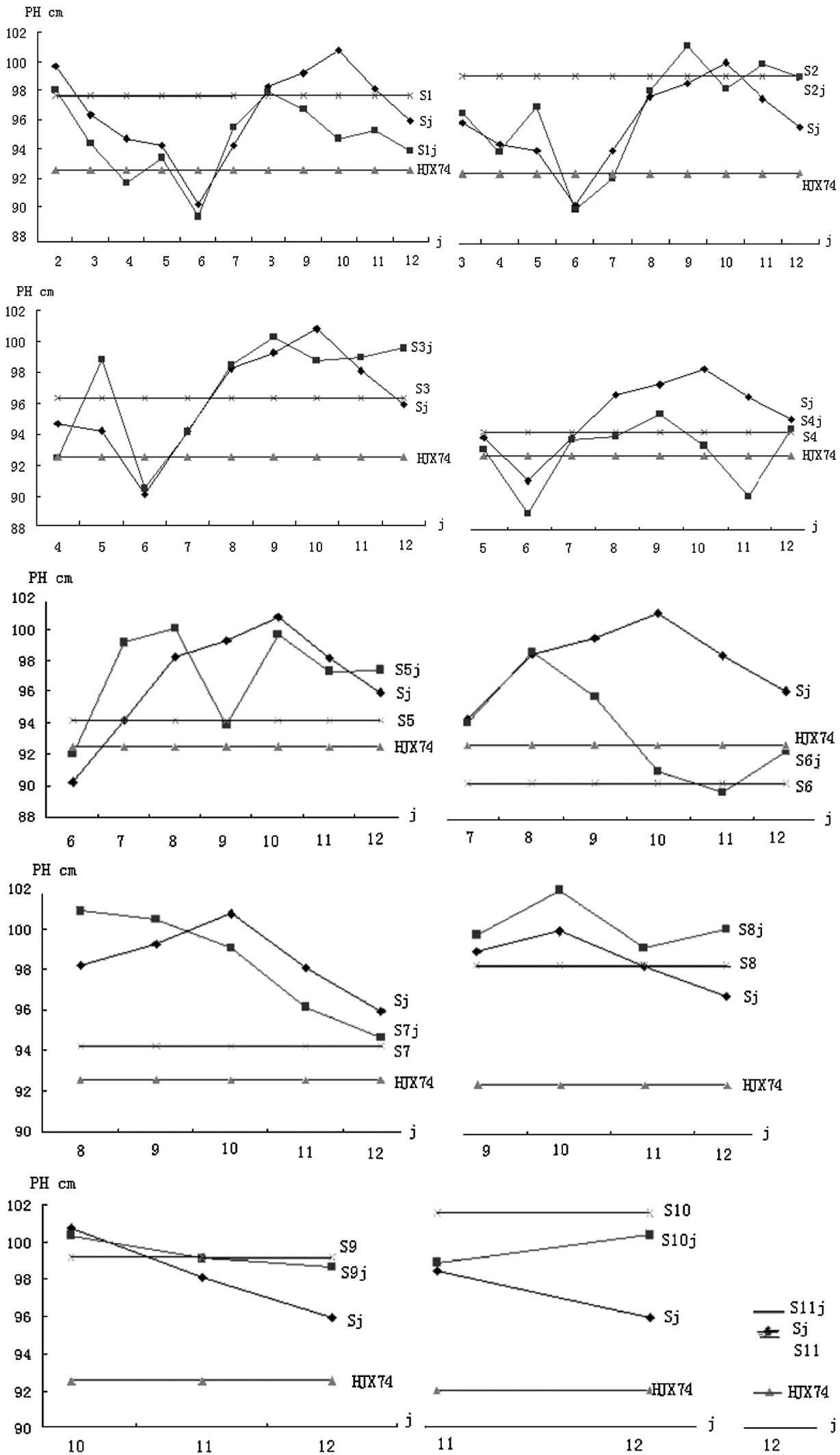

Fig. 1. Rough judgment of epistatic interactions by comparing average $\mathrm{PH}$ among different genotypes was suggested. The active points represented the genotypic values of HJX74, $\mathrm{SSSL}_{\mathrm{i}} \times \mathrm{HJX74}(\mathrm{Si}), \mathrm{SSSL}_{\mathrm{j}} \times \mathrm{HJX74}(\mathrm{Sj})$ and $\mathrm{SSSL}_{\mathrm{i}} \times \mathrm{SSSL}_{\mathrm{j}}(\mathrm{Sij})$ (here $i$ was not equal to $j$ ) at each QTL, respectively. If the distance of points between Sij and $\mathrm{Sj}$ differed apparently from that between Si and HJX74, the digenic interaction could exist between $\mathrm{QTL}_{\mathrm{i}}$ and QTL $\mathrm{L}_{\mathrm{j}}$. For example, since the distance between S1.10 and S10 kept nearly consistent with that between S1 and HJX74, it implied that QTL on S1 was independent of QTL on S10. In the contrary, QTL on S1 interacted with other QTLs since the distances differed apparently. 
detected with significant epistatic effects under $\mathrm{d} 1, \mathrm{~d} 2$ and d3, respectively. These results indicated that high density conditions perhaps promoted digenic interaction on $\mathrm{PH}$ in rice. Moreover, epistatic effects estimated changed across the three planting densities. Although QTL pairs exhibited mostly consistent effect directions e.g. negative epistatic interactions, DES calculated among the densities changed from $1.4 \mathrm{~cm}$ to $26.6 \mathrm{~cm}$ (Table 2). Some epistatic effects even appeared different interaction directions across the densities. The results suggested that epistasis perhaps was one of causes to produce phenotypic variation of $\mathrm{PH}$ among the densities, and that epistatic interactions on PH QTLs had intense density sensitivity.

\section{Discussion}

In present study, the analyses of epistatic interactions among QTLs on plant height in rice were performed using 12 SSSLs. The results provided concrete evidence for the existence of epistatic interactions among them, and revealed that these QTLs were involved in planting density sensitivity. Our previous studies (Liu et al. 2008, 2009, 2010, 2012) confirmed that SSSLs are ideal materials to detect QTLs and analyze interactions between QTLs and environments. Our recent study (Liu et al. 2012) and this paper more clearly indicated that the use of SSSLs is a powerful and effective method to detect and characterize QTL epistatic interaction. Although QTL-NILs provide a number of advantages for the study of QTLs, construction of QTL-NILs is labor intensive and takes a long time (Ashikari and Matsuoka 2006). On the other hand, the use of SSSL library should be more effective for searching and identifying target QTLs (Keurentjes et al. 2007). Although SSSLs possess relatively large donor chromosome segments compared with QTLNILs, their genetic constitutions can be easily manipulated to generate shorter introgressions that retain the phenotypic effects. In our lab, a mass of SSSLs with donor segments less than $10 \mathrm{cM}$ have successfully been achieved and they are going to play a major role for QTL analysis and QTL pyramiding for breeding (Wang et al. 2012). In our previous studies, we employed SSSLs to identify and character additive, dominance, epistasis and their interaction effects with environments of QTLs on tiller number and panicle number per plant (Liu et al. 2008, 2009, 2010, 2012). In this study 12 SSSLs were sequentially selected as experimental materials with the aim to detect and character epistatic interactions among QTLs on plant height per plant in rice.

For the analysis of epistasis, a diallel crossing scheme can be used to generate heterozygotes or homozygotes containing a number of introgressions that are analyzed in a simple experiment with balanced representation of the different genotypes. The introgressions can be combined in different genetic constitutions enabling an analysis of all possible interactions between the components of the genetic variation (Eshed and Zamir 1996, Hua et al. 2003). To analyze epistatic interaction between pairs of QTLs, for instance, we can pyramid a dual segment substitution line (DSSL) via MAS from the F2 population of two SSSL crossing, and then construct a half diallel crossing population from four parents (including recipient, two SSSLs and DSSL) to generate 9 genotypes consisting of two QTLs (Table 3). We can investigate target traits under various experimental conditions, and then estimate QTL effects based on phenotypic data recorded on the all genotypes. The genetic model for the analysis is $D_{i j}=S_{i}+S_{j}+S_{i} \times S_{j}$, where $S_{i}, S_{j}, D_{i j}$ are the genotypic effects of introgressions from the control (like HJX74), which significances are tested by the least significant difference (LSD) method with the statistics $L S D_{\alpha}=t_{a} \sqrt{\frac{2 M S_{e}}{n}}$. Where $M S_{e}$ is the experimental error with the degree of freedom $d f_{e}=k(n-1), k, n$ are the numbers of participating genotypes and replications respectively, and $t_{a}$ is the critical $t$-value with $d f_{e}$ degree of freedom and $\alpha$ probability. Then the interaction effects can be estimated as $S_{i} \times S_{j}=D_{i j}-S_{i}-S_{j}$. For instance, for the genotypes of $A A B B, A A B b, A a B B$ and $A a B b$, their epistatic effects can expressed as $e_{a-a}=D_{i j}-a_{i}-a_{j}, e_{a-d}=D_{i j}-a_{i}-$ $d_{j}, e_{d-a}=D_{i j}-d_{i}-a_{j}$ and $e_{d-d}=D_{i j}-d_{i}-d_{j}$, representing

Table 3. Construction of a mapping population of a half diallel crossing from four parents

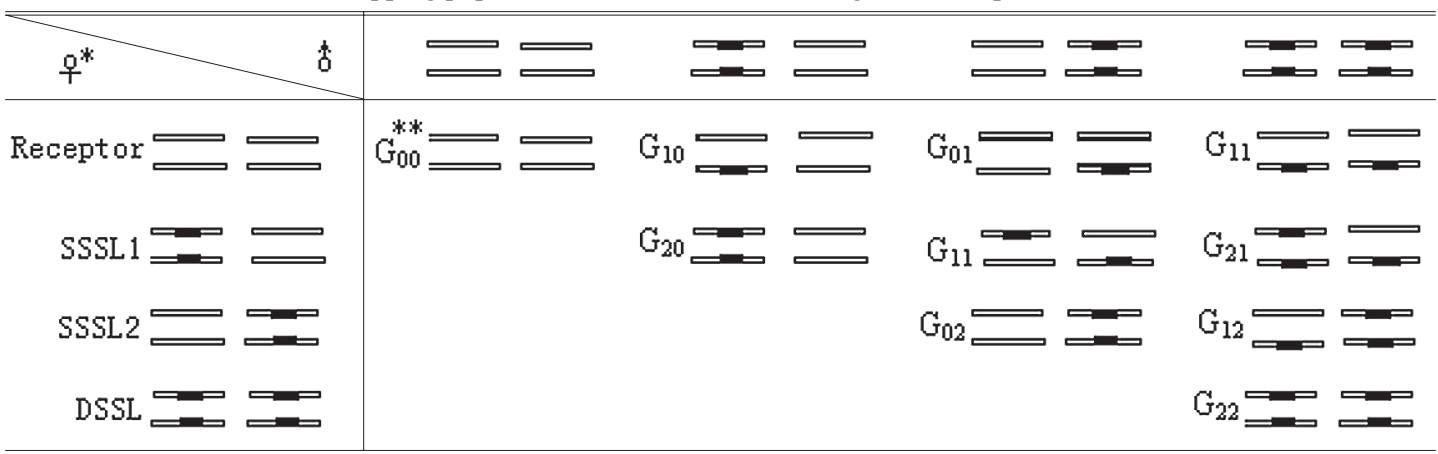

* SSSL and DSSL represent single and dual segment substitution lines, respectively.

** $G_{i j}$ is genotype at loci $i$ and $j(i, j=0,1,2) . G_{00}, G_{20}, G_{02}$ and $G_{22}$ represent four parents of receptor, SSSL1, SSSL2 and DSSL. 
four types of epistatic interactions between the two QTLs, e.g. additive-additive, additive-dominance, dominanceadditive and dominance-dominance epistasis, respectively. Their significances can still be tested by LSD method with $L S D_{\alpha}=t_{a} \sqrt{\frac{4 M S_{e}}{n}}$ since these estimations amount to $e_{i j}=$ $\left(D S S L_{i j}+H J X 74\right)-\left(S S S L_{i}+S S S L_{j}\right)$, where all components on the right of the equation represent phenotypic mean values of corresponding genotypes. Adopting this method, Eshed and Zamir (1996) and Lin et al. (2000) estimated dominance-dominance and additive-additive epistatic effects between QTLs, respectively. We also ever reported QTL dominance-dominance epistatic interaction (Liu et al. 2012). Again this paper analyzed dominance-dominance epistasis of QTLs using this method. However, no studies can simultaneously provide the information about the four types of digenic interactions so far. In this paper since absence of genotypes like $A A B B, A A B b, A a B B$, we just can estimate the dominance-dominance epistatic interaction from $A a B b$. Further for three QTLs, there are additional 8 types of epistatic interactions besides digenic QTLs. The genetic model for the analysis is $T_{i j k}=S_{i}+S_{j}+S_{k}+S_{i} \times S_{j}$ $+S_{i} \times S_{k}+S_{j} \times S_{k}+S_{i} \times S_{j} \times S_{k}$, where $T_{i j k}$ is the genotypic effect of three-segment introgressions. Thus the interaction effects among three QTLs can be estimated as $S_{i} \times S_{j} \times S_{k}=$ $T_{i j k}-S_{i}-S_{j}-S_{k}-S_{i} \times S_{j}-S_{i} \times S_{k}-S_{j} \times S_{k}$, where all components on the right of the equation can be determined by the method aforementioned. Trigenic interaction can be tested by $L S D_{\alpha}=t_{a} \sqrt{\frac{8 M S_{e}}{n}}$. Similarly for more QTLs, we can extend this methodology to infer epistatic interaction among multi QTLs.

At least 1011 QTLs for PH in rice have been saved in Gramene QTL Database (http://www.gramene.org/qtl/). Nevertheless, one of the functions of these QTLs, response to density environments, still remains obscure. In this paper 12 QTLs on PH were confirmed, and some of them exhibited environmental sensitivity to planting density. A significant finding was that $\mathrm{d} 2$ density conditions would inhibit the expressing of these PH QTLs. Epistatic interactions of $\mathrm{PH}$ QTLs, as one of important genetic components, have few been reported yet. Although there were extensive evaluations in primary populations (such as $\mathrm{F} 2, \mathrm{BC} 1$, or RIL populations), these populations cannot provide much information about the real nature of epistatic interactions among individual QTLs (Yano et al. 1997). By means of QTL-NILs, epistatic interactions were detected with higher frequencies and their functions were also analyzed well for several quantitative traits. Eshed and Zamir (1996) reported that for five yield-associated traits in tomato there were $20-42 \%$ of the 45 dichromosome segment combinations to be epistatic $(\mathrm{P}<0.05)$. Lin et al. (2000) used QTL-NILs to have detected epistatic interactions among $H d 1, H d 2$ and $H d 3$ on heading date in rice, one of which couldn't be detected using an F2 population. Moreover, they found that the epistatic interactions between $H d 1$ and $H d 2$ and between $H d 1$ and $H d 3$ were different under short daylength and long daylength or nature daylength conditions. In a our previous study, we used 12 SSSLs to analysis dynamics of QTLs on tiller number, in which about $89 \%$ of the 66 dichromosome segment combinations involved in dominance-dominance epistatic interactions at 7 investigated stages and these epistatic effects were sensitive to growing seasons and planting densities. In this paper above phenomenon were reappeared on the 12 PH QTLs. Most QTL pairs exhibited epistatic interactions, but they can be detected just at certain conditions and appeared sensitivity to planting densities. It seemed that high density conditions promoted to detect epistatic interactions between pairs of PH QTLs. All these results clearly showed that epistatic interactions between pairs of QTLs were prevalent and environment sensitive. In this paper to qualitatively analyze epistatic interactions among alleles at the 12 PH QTLs, we adopted a point figure to judge whether the epistasis existed or not (Fig. 1). This figure told us that epistatic interaction was possible to exist between two QTLs if the simple effect of $S_{i j}-S_{j}$ is significantly different from that of $S_{i}-H J X 74$. Subsequently via a two-way ANOVA we estimated quantitatively these epistatic effects (Table 2). A consistent trend was that these QTL interactions were mostly negative, whereby the phenotypic effects of the double heterozygotes were of a lower magnitude than the sum of the effects of the single heterozygotes. This interaction would be confounded with the effect of the individual QTL and then would result in over-estimating single locus effects. Negative epistatic interactions were detected on some yield-associated traits in tomato (Eshed and Zamir 1996) and on heading date in rice under nature daylength and long daylength conditions (Lin et al. 2000). We also found the consistent phenomena occurred on 12 tiller number QTLs in rice (Liu et al. 2012). This trend was more pronounced for three even more QTLs than for pairs of QTLs (Eshed and Zamir 1996, Lin et al. 2000). As a consequence, the comprehensive effect of multi-QTLs would be much less than the cumulative effect of these individual QTL. This feature of epistatic interaction maybe is advantageous to achieve diminishing target traits when performing molecular pyramiding breeding, but mostly negative epistatic interaction would obstruct improvement of target traits. However, when two QTLs with negative effects or opposite effects combined into an introgression, positive epistatic interactions would occur following the calculation $S_{i} \times S_{j}$ $=D_{i j}-S_{i}-S_{j}$. These phenomena need be confirmed and explained by experimental data and genetic principles, and how to utilize epistatic interactions among QTLs is a worthy topic to explore.

\section{Acknowledgments}

This research was supported by Guangdong Natural Science Foundation, China (S2013010012939 and S2013010013184), Guangzhou Science and Technology Planning Project Foundation, China (2014J4100241), the National Natural Science 
Foundation of P.R. China (31200905) and State Key Laboratory for Conservation and Utilization of Subtropical Agrobioresource Open Foundation, South China Agricultural University, China (SKL-CUSAb-2013-07).

\section{Literature Cited}

Ashikari, M. and M. Matsuoka (2006) Identification, isolation and pyramiding of quantitative trait loci for rice breeding. Trends Plant Sci. 11: 344-350.

Cho, Y., H.Kang, J.Lee, Y.Lee, S.Lim, H.Gauch, M.Eun and S. McCouch (2007) Identification of quantitative trait loci in rice for yield, yield components, and agronomic traits across years and locations. Crop Sci. 47: 2403-2417.

Cockerham, C. and Z.Zeng (1996) Design III with marker loci. Genetics 143: 1437-1456.

Cooper, M. and G. Hammer (1996) Plant adaptation and crop improvement. CAB International, Oxon.

Eshed,Y. and D.Zamir (1995) An introgression line population of Lycopersicon pennellii in the cultivated tomato enables the identification and fine mapping of yield-associated QTL. Genetics 141: $1147-1162$.

Eshed, Y. and D. Zamir (1996) Less-than-additive epistatic interactions of quantitative trait loci in tomato. Genetics 143: 1807-1817.

Falconer, D. (1989) Introduction to Quantitative Genetics. John Wiley and Sons, New York.

Gao, Y. and J.Zhu (2007) Mapping QTLs with digenic epistasis under multiple environments and predicting heterosis based on QTL effects. Thoer. Appl. Genet. 115: 325-333.

He, F., Z.Xi, R.Zeng and G.Zhang (2005a) Developing single segment substitution lines (SSSLs) in rice (Oryza sativa L.) using advanced backcrosses and MAS. Acta Genet. Sin. 32: 825-831.

He, F., Z.Xi, R. Zeng, A. Talukdar and G. Zhang (2005b) Mapping of heading date QTLs in rice (Oryza sativa L.) using single segment substitution lines. Acta Agric. Sin. 38: 1505-1513.

He, F., R.Zeng, Z.Xi, A. Talukdar and G. Zhang (2005c) Identification of QTLs for plant height and its components by using single segment substitution lines in rice (Oryza sativa L.). Rice Sci. 12: $151-156$.

Hua, J., Y.Xing, W.Wu, C.Xu, X.Sun, S.Yu and Q.Zhang (2003) Single-locus heterotic effects and dominance by dominance interactions can adequately explain the genetic basis of heterosis in an elite rice hybrid. Proc. Natl. Acad. Sci. USA 100: 2574-2579.

Keurentjes, J., L. Bentsink, C. Alonso-Blanco, C. Hanhart, V. BlankestijnDe, S. Effgen, D. Vreugdenhil and M.Koornneef (2007) Development of a near-isogenic line population of Arabidopsis thaliana and comparison of mapping power with a recombinant inbred line population. Genetics 175: 891-905.

Li, L., K. Lu, Z. Chen, T. Mu, Z. Hu and X. Li (2008) Dominance, overdominance and epistasis condition the heterosis in two heterotic rice hybrids. Genetics 180: 1725-1742.

Li,Z., S.Pinson, W.Park, A.Paterson and J.Stansel (1997) Epistasis for three grain yield components in rice (Oryza sativa L.). Genetics 145: 453-465.

Li,Z., L. Luo, H. Mei, D. Wang, Q. Shu, D. Wang, R. Tabien, D.Zhong, C. Ying, J. Stansel et al. (2001) Overdominant epistatic loci are the primary genetic basis of inbreeding depression and heterosis in rice. I. Biomass and grain yield. Genetics 158: 1737-1753.

Li,Z., S.Yu, H.Lafitte, N.Huang, B.Courtois, S. Hittalmani, C. Vijayakumar, G. Liu, G. Wang, H. Shashidhar et al. (2003) QTL $\times$ environment interactions in rice. I. Heading date and plant height. Theor. Appl. Genet. 108: 141-153.

Lin, H., T. Yamamoto, T. Sasaki and M. Yano (2000) Characterization and detection of epistatic interactions of 3 QTLs, $H d 1, H d 2$ and $H d 3$, controlling heading date in rice using nearly isogenic lines. Thoer. Appl. Genet. 101: 1021-1028.

Liu, G., Z.Zhang, H.Zhu, F.Zhao, X.Ding, R.Zeng, W.Li and G.Zhang (2008) Detection of QTLs with additive effects and additive-by-environment interaction effects on panicle number in rice (Oryza sativa L.) with single-segment substitution lines. Theor. Appl. Genet. 116: 923-931.

Liu, G., R.Zeng, H.Zhu, Z.Zhang, X.Ding, F.Zhao, W.Li and G. Zhang (2009) Dynamic expression of nine QTLs for tiller number detected with single segment substitution lines in rice. Theor. Appl. Genet. 118: 443-453.

Liu, G., H.Zhu, S. Liu, R.Zeng, Z.Zhang, W.Li, X. Ding, F.Zhao and G.Zhang (2010) Unconditional and conditional QTL mapping for the developmental behavior of tiller number in rice (Oryza sativa L.). Genetica 138: 885-893.

Liu, G., H.Zhu, G.Zhang, L. Li and G. Ye (2012) Dynamic analysis of QTLs on tiller number in rice (Oryza sativa L.) with single segment substitution lines. Theor. Appl. Genet. 125: 143-153.

Ming, S. (1987) Breeding of semi-dwarf rice. In: Yuang, S.R. (ed.) Rice, China Agriculture Press, Beijing, pp. 66-67.

Paterson,A.H., S.Damon, J.D.Hewitt, D.Zamir, H.D. Rabinowitch, S.E. Lincoln, E.S. Lander and S.D. Tanksley (1991) Mendelian factors underlying quantitative traits in tomato: comparison across species, generations, and environments. Genetics 127: 181-197.

SAS Institute (1988) SAS users guide: statistics. SAS Institute, Cary, N.C.

Semel, Y., J. Nissenbaum, N. Menda, M.Zinder, U. Krieger, N. Issman, T. Pleban, Z.Lippman, A. Gur and D.Zamir (2006) Overdominant quantitative trait loci for yield and fitness in tomato. Proc. Natl. Acad. Sci. USA 103: 12981-12986.

Tanksley, S. (1993). Mapping polygenes. Annu. Rev. Genet. 27: 205233.

Tian, F., D. Li, Q.Fu, Z.Zhu, Y.Fu, X. Wang and C.Sun (2006) Construction of introgression lines carrying wild rice (Oryza rufipogon Griff.) segments in cultivated rice (Oryza sativa L.) background and characterization of introgressed segments associated with yield-related traits. Theor. Appl. Genet. 112: 570-580.

Wan, J., H.Zhai, J.Wan, H. Yasui and A. Yoshimura (2003) Mapping QTL for traits associated with resistance to ferrous iron toxicity in rice (Oryza sativa L.), using japonica chromosome segment substitution lines. Acta Genet. Sin. 30: 893-898.

Wang,D., J.Zhu, Z.Li and A.Paterson (1999) Mapping QTLs with epistatic effects and QTL $\times$ environment interactions by mixed linear model approaches. Theor. Appl. Genet. 99: 1255-1264.

Wang, S., K.Wu, Q.Yuan, X.Liu, Z.Liu, X.Lin, R.Zeng, H.Zhu, G. Dong, Q. Qian et al. (2012) Control of grain size, shape and quality byOsSPL16 in rice. Nat. Genet. 44: 950-954.

Xi,Z., F.He, R.Zeng, Z.Zhang, X. Ding, W.Li and G.Zhang (2006) Development of a wide population of chromosome single-segment substitution lines in the genetic background of an elite cultivar of rice (Oryza sativa L.). Genome 49: 476-484.

Xiao, J., J.Li, L. Yuan and S. Tanksley (1995) Dominance is the major genetic basis of heterosis in rice as revealed by QTL analysis using molecular markers. Genetics 140: 745-754.

Yamamoto, T., H.Lin, T. Sasaki and M. Yano (2000) Identification of heading date quantitative trait locus $H d 6$ and characterization of its epistatic interactions with $H d 2$ in rice using advanced backcross 
progeny. Genetics 154: 885-891.

Yano, M., Y.Harushima, Y.Nagamura, N.Kurata, Y.Minobe and T. Sasaki (1997) Identification of quantitative trait loci controlling heading date in rice using a high-density linkage map. Theor. Appl. Genet. 95: 1025-1032.

Yano, M., Y. Katayose, M.Ashikari, U. Yamanouchi, L. Monna, T. Fuse, T. Baba, K. Yamamoto, Y. Umehara, Y. Nagamura et al. (2000) Hd1, a major photoperiod sensitivity quantitative trait locus in rice, is closely related to the Arabidopsis flowering time gene CONSTANS. Plant Cell 12: 2473-2483.

Yano, M., S. Kojima, Y. Takahashi, H. Lin and T. Sasaki (2001) Genetic control of flowering time in rice, a short-day plant. Plant Physiol. 127: 1425-1429.

Zhang, G., R.Zeng, Z.Zhang, X.Ding, W.Li, G.Liu, F.He, A. Tulukdar, C.Huang, Z.Xi et al. (2004) The construction of a library of single segment substitution lines in rice (Oryza sativa L.). Rice Genet. Newsl. 21: 85-87.
Zhao, F., G.Liu, H.Zhu, X.Ding, R.Zeng, Z.Zhang, W.Li and G.Zhang (2008) Unconditional and conditional QTL mapping for tiller numbers at various stages with single segment substitution lines in rice (Oryza sativa L.). Agric. Sci. China 7: 257-265.

Zhao, X., Y.Qin and J.Sohn (2010) Identification of main effects, epistatic effects and their environmental interactions of QTLs for yield traits in rice. Genes Genomics 32: 37-45.

Zhao, X., Y.Qin, B.Jia, S. Kim, H.Lee, M.Eun, K. Kim and J.Sohn (2013) Comparison and analysis of QTLs, epistatic effects and QTL $\times$ environment interactions for yield traits using DH and RILs populations in rice. Journal of Integrative Agriculture 12: 198-208.

Zhu, J., Y.Zhou, Y.Liu, Z.Wang, Z.Tang, C.Yi, S.Tang, M. Gu and G. Liang (2011) Fine mapping of a major QTL controlling panicle number in rice. Mol. Breeding 27: 171-180.

Zhuang, J., H. Lin, J. Lu, H. Qian, S. Hittalmani, N. Huang and K. Zheng (1997) Analysis of QTL $\times$ environment interaction for yield components and plant height in rice. Theor. Appl. Genet. 95: 799-808. 